\title{
Magnetic Resonance Elastography : in vivo Measurements of Elasticity for Human Tissue
}

\author{
Takenori OIDA, Akira AMANO and Tetsuya MATSUDA \\ Graduate school of Informatics, Kyoto University \\ Yoshida Hon-machi, Sakyo-ku, Kyoto 606-8501 JAPAN \\ oida@sys.i.kyoto-u.ac.jp, \{amano, tetsu\}@i.kyoto-u.ac.jp
}

\begin{abstract}
Elasticity is an important physical property of material. In the clinical practice, elasticity is used for physical examination in several ways, such as palpation or percussion. Differences in elasticity can help facilitate the diagnosis of tumors and their extent. Elasticity is an essential property in the diagnosis of liver cirrhosis, or soft degeneration in tissue necrosis. In addition, information of tissue elasticity is utilized in virtual reality systems such as telepalpation and computer assisted surgery. It was difficult to obtain such properties in vivo by using conventional measurement methods. To overcome this problem, magnetic resonance elastography (MRE) has been developed that provides noninvasive in vivo measurements of elasticity for human tissue. We summarize this MRE method in this paper.

When an object is oscillated from the surface in a known frequency, acoustic strain waves propagate into the material and one can calculate the physical constants of a material elasticity by the wave velocity. In MRE measurements, a cyclic micromotion caused by the acoustic strain waves is obtained as an MR image that is synchronized to the oscillation. By measuring the local wavelength of the strain waves, we can obtain the elasticity constants. Several examples of MRE image including in vivo measurements are provided as well as several methods to estimate the local wavelength from MRE images are described in this paper.
\end{abstract}

\section{Introduction}

"Stiffness" is one of the important index for the characteristics of the materials. By touching and sensing the material whether they are "hard" or "soft", we can easily distinguish rigid materials such as steels or diamonds from elastic materials such as rubbers or jellies. In a similar sense, human tissues can also be distinguished as "hard" for bones or "soft" for various internal organs or skins. If the "tissue stiffness" can be measured noninvasively, we can ex- pect that they can be used as a new index for the diagnosis of the various diseases such as liver cirrhosis and tumor which become harder than normal tissue, or necrosis which is softer. In addition, information of tissue elasticity is an very important parameter for virtual reality systems such as telepalpation and computer assisted surgery that simulates tissue deformation with a finite element method or massspring model.

These physical properties concerning stiffnesses of material are called elasticity. Conventional measurement method requires to cut off a piece of material from the body and elastic properties were calculated by measuring the amount of deformations under certain external forces. Therefore, we could not measure tissue stiffness in vivo and in situ, and we could not use the "tissue stiffness" as a quantitative index of the diagnosis of the various diseases.

Recently, a new method called magnetic resonance elastography (MRE) was proposed by Mathupillai et al. of Mayo Clinic [1-5] that measured the elasticity of human tissue with MRI. This method is completely different from conventional methods in the sense that it is nondestructive, allowing us to measure human tissue elasticity in vivo in the clinical practice.

In this paper, we describe the principles of MRE method and the basic dynamics of the elastic materials, and then the principle of MRE method is explained. Finally, the characteristics of MRE method are illustrated with actual MRE images.

\section{Elasticity of a material}

The basic property of the elasticity is described by the "Hooke's law", which denotes that the amount of stretch of a spring is proportional to the applied force $[6,7]$. In other words Hooke's law is described as follows,

$$
F=k x
$$




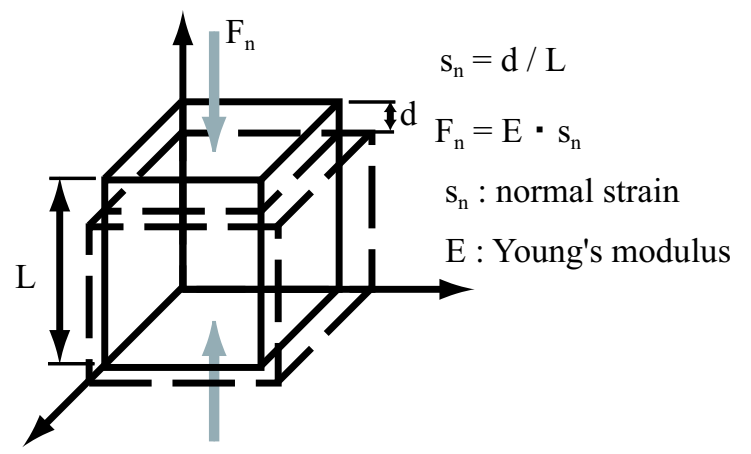

Figure 1. The relationship between the Young's modulus and the normal strain.

where $F$ stands for the applied force, and $x$ stands for the stretch of spring, and $k$ is the spring constant.

Instead of spring, consider an elastic cube of the height $L$ as shown in Figure 1. When it is compressed with the force $F_{n}$ per unit area, which is called stress, the height shortens by distance $d$. The $s_{n}$ defined as $s_{n}=d / L$ is called strain. If the strain is small, the relationship between stress $F_{n}$ and strain $s_{n}$ follows Hooke's law:

$$
F_{n}=E \cdot s_{n}
$$

The proportional constant $E$ is called Young's modulus, which is one of the elastic constants and it indicates the stiffness of a material.

Another important elastic constant is rigidity, which is also called as shear modulus. It represents the deformation of a material under a force whose direction is parallel to the surface of material. Consider an elastic cube of the height $H$ (Figure 2). When a force is applied in the direction parallel to the top surface, the position of the top surface moves by distance $d$. The $s_{s}$ defined as $s_{s}(=d / H)$ is called shear strain. The relationship between shear stress $F_{s}$ and shear strain $s_{s}$ also follows Hooke's law:

$$
F_{s}=G \cdot s_{s}
$$

This proportional constant $G$ is called shear modulus.

In addition, bulk modulus $K$ and Poisson's ratio $\sigma$ are also defined as elastic constants of a material. They describe the elasticity relating to the compression and the threedimensional strain of the material. There are only two degree of freedom for those constants, thus only two of them are independent. This means that the arbitrary two elastic constants can be calculated from the other two constants. For example, by using the Young's modulus $E$ and the shear modulus $G$, the bulk modulus $K$ and the Poisson's ratio $\sigma$ can be calculated as follows.

$$
K=\frac{E G}{9 G-3 E}
$$

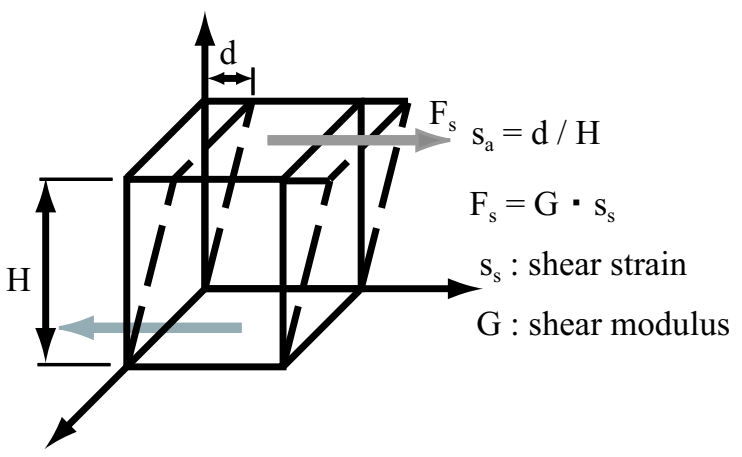

Figure 2. The relationship between the shear modulus and the shear strain.

$$
\sigma=\frac{E-2 G}{2 G}
$$

MR elastography is a method which calculates shear modulus and Young's modulus by measuring the waves caused by oscillating the material externally.

When the several oscillations are applied to the material, they cause the acoustic strain waves and they propagate into the deeper part of the material. There are two types in the acoustic strain waves, one is the longitudinal wave and the other is the transverse wave. Figure 3 and Figure 4 show the strain wave propagations in an elastic body. When the shear stresses are applied to a top surface of a hard material such as steel, shear strains propagate to the bottom immediately. If oscillations of these shear stresses are applied with a fixed frequency, the strain waves propagate to the bottom immediately (Figure 3). In other words, the wavelength of the strain wave is very long, and the phase of wave at the bottom is almost the same as that at the top. On the other hand, in the case of a soft material such as rubber, shear stresses propagate into the bottom slowly. When oscillations of the shear stress are applied, the velocity of strain waves are slow. The wavelength is short and the oscillations propagate as reciprocating motion in the material (Figure 4). Such discussion can be also applied to the case of longitudinal waves where the propagation velocity becomes much large.

The relationship between the propagating velocity of acoustic strain waves and the elastic constants are described as follows.

$$
\begin{aligned}
& v_{t}=\sqrt{\frac{G}{\rho}} \\
& v_{l}=\sqrt{\frac{G(4 G-E)}{\rho(3 G-E)}}
\end{aligned}
$$




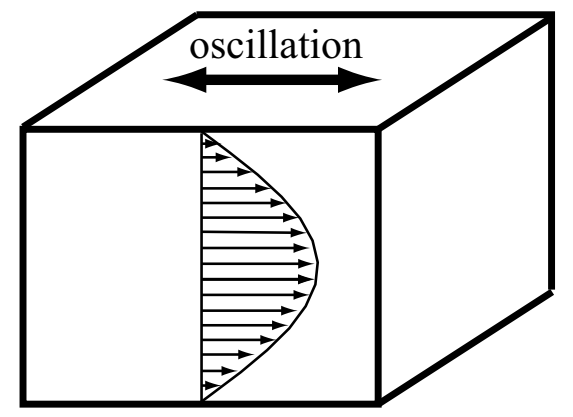

Figure 3. Propagation of the acoustic strain waves in a hard material. The acoustic strain waves propagate rapidly in a hard material and the wavelength is long.

Note that, $E$ is Young's modulus, $G$ is shear modulus, $\rho$ is density of the material, respectively. The $v_{t}$ and $v_{l}$ are velocities of the transverse and the longitudinal waves.

With the bulk modulus $K$ and the shear modulus $G$, the velocity $v_{l}$ can be described as follows.

$$
v_{l}=\sqrt{\frac{K+4 G / 3}{\rho}}
$$

Comparing eq.(1) with eq.(3), we can see that a longitudinal wave is faster than a transverse wave because of $v_{t}<v_{l}$.

As the velocity $v$ of the wave can be described with the frequency $f$ of the oscillation and the wavelength $\lambda$ as $v=$ $f \lambda$, eq.(1) and eq.(2) can be represented as follows.

$$
\begin{aligned}
& G=\rho\left(f_{t} \lambda_{t}\right)^{2} \\
& E=\rho \frac{3 \rho G\left(f_{l} \lambda_{l}\right)^{2}-4 G^{2}}{\rho G\left(f_{l} \lambda_{l}\right)^{2}-G}
\end{aligned}
$$

where $f_{t}, \lambda_{t}, f_{l}, \lambda_{l}$ represents the frequency and the wavelength of transverse and longitudinal waves, respectively.

In MR elastography method, external oscillations are applied to a material with known frequency. The acoustic strain waves caused by the oscillations are visualized in MR images. Thus, the shear modulus and the Young's modulus are calculated from eq.(4) and eq.(5) by measuring the wavelength from the images. For the accurate measurement, it is necessary to measure the density $\rho$ of a material. However, in the case of human tissue, it can be approximated as $\rho \approx 1.0$.

\section{The basic principles of MR elastography}

In this section, the basic principles to acquire the propagating waveforms inside the materials from images of MRE

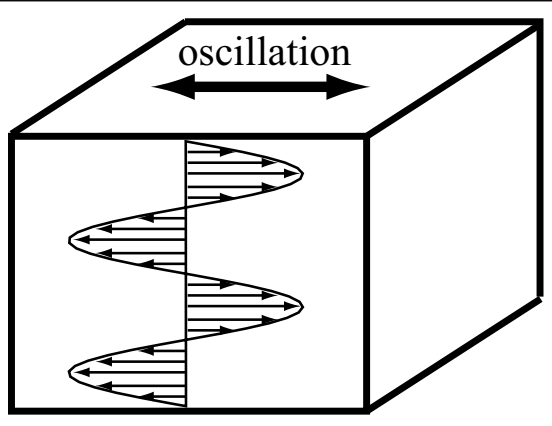

Figure 4. Propagation of acoustic strain waves in a soft material. When the material is soft, velocity of the acoustic strain waves is slow and the wavelength is short.

are described.

When the surface of a material is oscillated with certain frequency, the strain waves are propagating into it and every point in the material reciprocates in micromotion. MRI has high sensitivity to the motions and MRE utilizes this feature to acquire waveforms.

The motion sensitive methods for MRI are classified to phase shift method and time-of-flight method, which are used for quantification of velocity or MR angiography in the clinical practice. However, such conventional velocity imaging methods do not have enough sensitivity for micromotion. While phase shift methods [8,9] have higher sensitivity for the slow velocity motions than the time-of-flight methods, they don't have enough sensitivity to image the strain waves. To overcome this problem, MRE method exploites the cyclic feature of the micromotion to increase the sensitivity of the phase shift method.

Figure 5 shows an example of MR elastography pulse sequence which is reported by Muthpillai et al. [1]. This sequence is a kind of the standard gradient echo sequences, to which the gradient magnetic field called MSG (motion sensitizing gradient) is added. MSG is a series of oscillating polarity gradients in the read-out direction applied before the signal acquisition. This pulse sequence is similar to the phase contrast imaging of MR angiography or velocity measurement MR imaging, in which only a single gradient magnetic field is applied. Therefore, we can say that the MSG is the essence of MRE method. The important point is that the switching of the MSG polarity is synchronized to the external force oscillation.

Consider that an arbitrary point in a elastic object is oscillating in right to left direction as in Figure 6 and a MSG is applied to the same direction. When the point starts moving to the right at time $t_{a}$, we carefully apply the first positive MSG. In this moment, small phase shift occurs until 


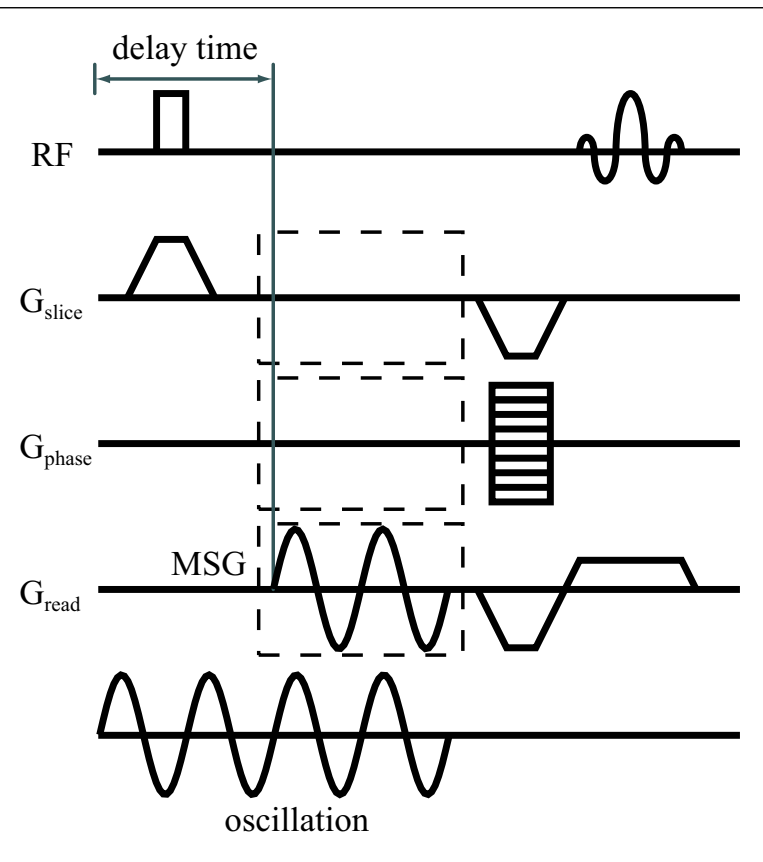

Figure 5. An example of MR elastography pulse sequence with gradient echo acquisition. MSG (motion sensitizing gradients) encodes the fine oscillation induced by acoustic strain waves into the phase of the acquired NMR signal.

time $t_{b}$ by the micromotion towards right. In the following time period, the point moves backward to the left. In this moment, the polarity of the MSG is switched to the reverse direction, thus the resulting polarity of the phase shift derived form the negative MSG and the motion of negative direction becomes the same as previous time period. With the several repetition of this sequence, we can acquire an accumulated phase shift induced by the micromotion. After two acquisitions of MRE images with opposite polarity of MSG, phase subtraction was made between those two images to produce phase image. In the phase image, the intensity of each pixel represents the direction and the amount of motion velocity. Consequently, the strain wavelength can be obtained from phase image.

Though the micromotion can be visualized by using the MSG which is synchronized to the external force oscillation, the frequency of the oscillation is limited by the performance of the MSG switching speed. When we use gradient coils with the performance of realizing echo-planar imaging to generate MSG, the minimum ramp time of the gradients is about $0.2 \mathrm{~ms}$ to $0.3 \mathrm{~ms}$. When the ramp time of $0.25 \mathrm{~ms}$ is used for MSG, one MSG cycle becomes $1 \mathrm{~ms}$ and the frequency of the oscillation is limited to $1000 \mathrm{~Hz}$. For

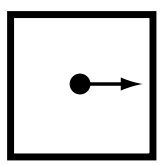

$t_{a}$

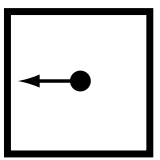

$\mathrm{t}_{\mathrm{b}}$

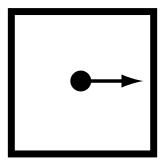

$t_{c}$

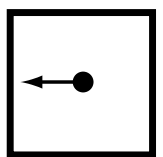

$t_{d}$

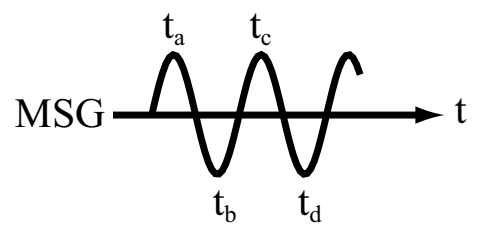

Figure 6. Motion sensitizing gradients (MSG) and the local oscillation. Since MSG is gated to oscillation, the direction of motion always agrees with polarity of gradients. The resulting phase shifts are amplified by MSG.

this reason, the oscillation frequency of several hundreds $\mathrm{Hz}$ are used in MRE. Practically, several cycles of MSG with strong gradients from $20 \mathrm{mT} / \mathrm{m}$ to $25 \mathrm{mT} / \mathrm{m}$ is used for MRE. In these days, the performance of gradient coils is improved to generate stronger strength and faster gradient switching in order to realize the echo-planar methods. With such improvement, MRE method can be realized.

\section{Actual MRE method}

By using an external force oscillator which synchronizes to the MSG of MRE pulse sequence, we can acquire MRE images. MRE acquisition system which we employs is shown in Figure 7, and its actuator is shown in Figure 8. In this system, the MRI controller generates trigger signal that is synchronized with the pulse sequence program. By receiving this trigger signal, waveform synthesizer starts to drive the actuator to apply oscillation to the material. A trigger signal produces several oscillations whose direction is parallel to the top surface of the material (Figure 8). Then, the MRI controller turns on the MSG after a certain delay time (one or several cycles). This actuator makes transverse waves, and we can calculate shear moduli from the acquired image representing the propagating transverse wave. Young's moduli can be acquired with application of propagating longitudinal wave, if the oscillator produces several oscillations perpendicular to the top surface of a material.

Examples of MRE images with transverse and longitudinal strain waves under $125 \mathrm{~Hz}$ oscillation are shown in Figure 9. These images are acquired with the phantom which is made from 5\% poly vinyl alcohol (PVA) hydrogel. The wavelength of longitudinal strain waves is longer than that of transverse strain waves in spite of the same oscilla- 


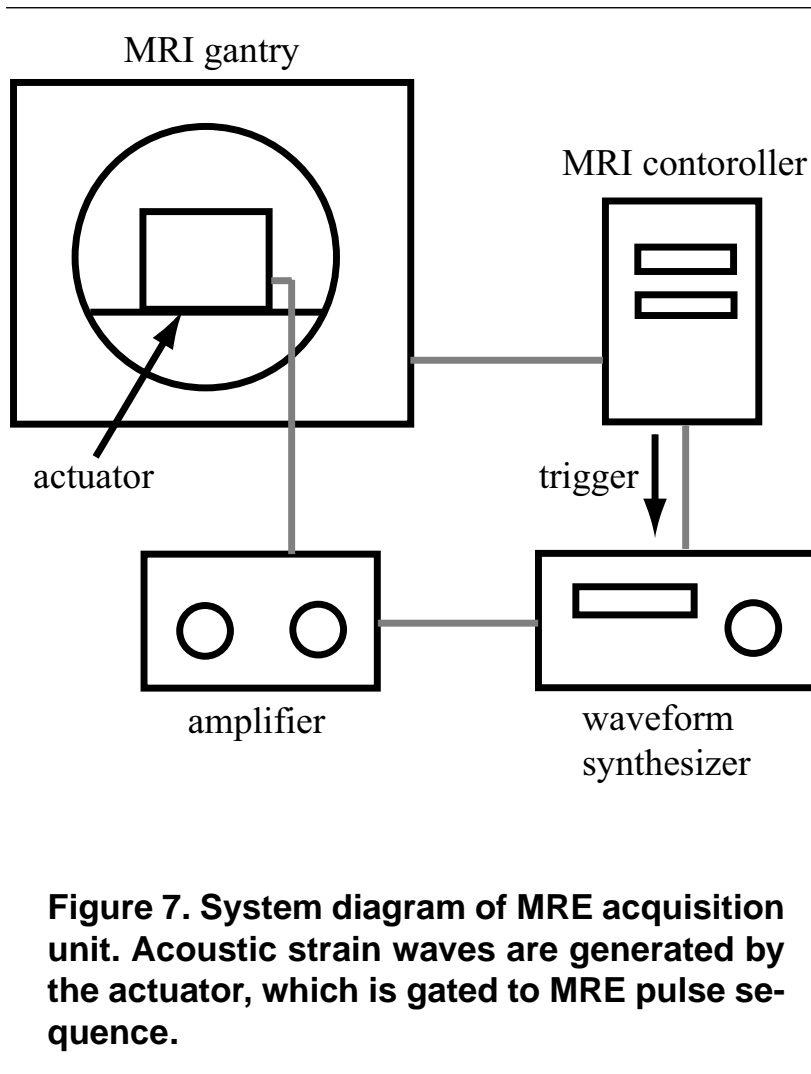

tion frequency, representing that the velocity of longitudinal strain wave is faster than that of transverse strain wave. On the other hand, in both images, acoustic strain waves become unclear along with the propagation, which shows the attenuation of the waves.

When the concentration of PVA hydrogel is increased, its stiffness becomes harder. We made a two layered PVA hydrogel phantom with different concentrations to show that MRE images can demonstrate the difference of stiffness. The left panel in Figure 10 shows T2-weighted image of the phantom whose top half is made from $10 \%$ PVA and bottom half is made from 5\% PVA. The top half is harder than the bottom. The right panel in Figure 10 shows an MRE image of this phantom obtained with application of $125 \mathrm{~Hz}$ transverse waves. The wavelength in the top half is longer than that in the bottom. This difference represents that the shear modulus $G$ in the top half is larger than that in the bottom from eq.(4), since the oscillation frequencies are the same.

Although we cannot see clearly in this image, reflection occurs at the boundary surface between two materials of different stiffness. Forward and reflection waves interfere each other at the area above the boundary. When a boundary surface is not perpendicular to the wave direction, not only the reflected wave but also the refracted wave will be generated. In that case, both longitudinal wave and transverse wave arise and the estimation of the wavelength becomes
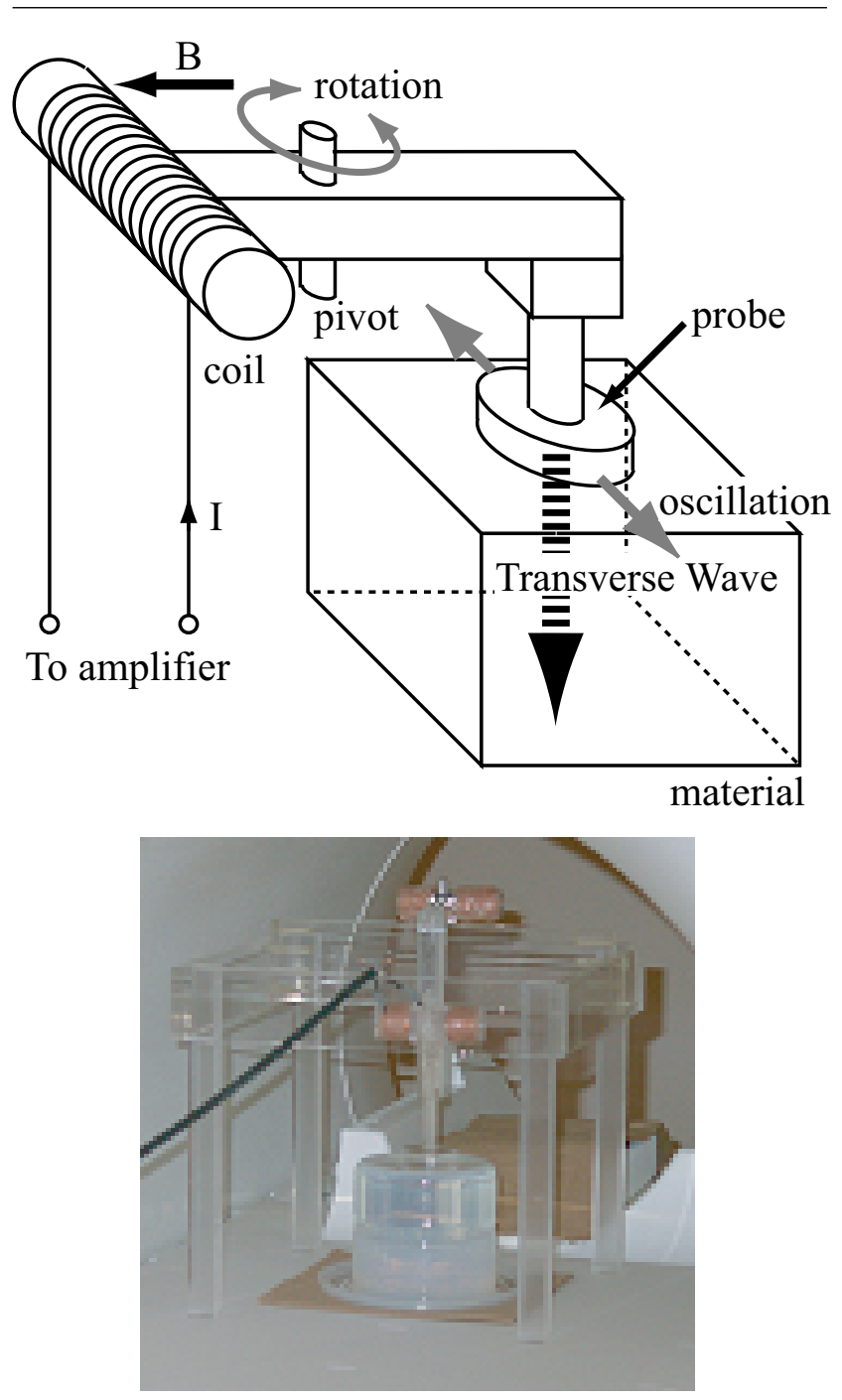

Figure 8. Diagram and an example of actuator. This actuator is implemented to be utilized in a magnetic field.

much complicated. The estimation of the wavelength from MRE images which include complex reflections and refractions is one of the most important problems in realizing clinical practice of MRE.

As we can easily expect, the appearance of wave in MRE image changes with the frequency of the oscillation. The velocity of the strain waves is determined by the Young's modulus and the shear modulus as shown in the eq.(1) and eq.(2). The velocity of the strain waves is a product of the frequency and the wavelength. Therefore, the wavelength of a material is proportional to the inverse of the oscillation frequency. Figure 11 shows MRE images of 7.5\% PVA 


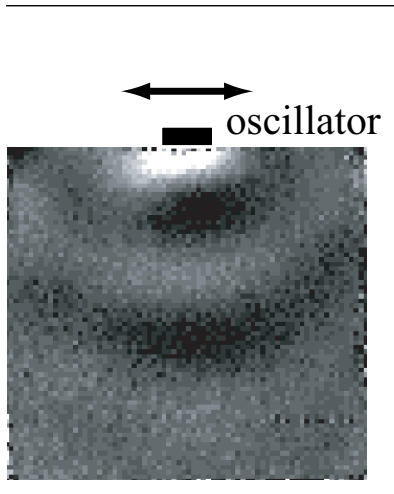

transverse waves

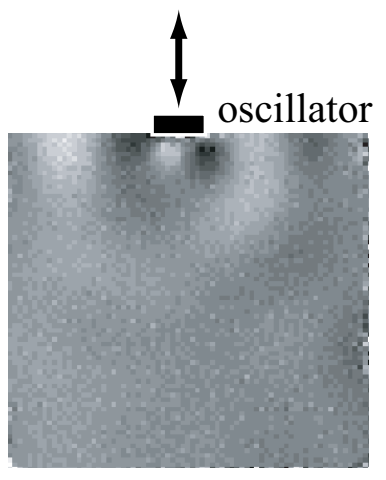

longitudinal waves
Figure 9. MRE of transverse and longitudinal strain waves. The wavelength of longitudinal strain waves is longer than that of transverse strain waves. Acoustic strain waves become unclear along with the propagation in both images.

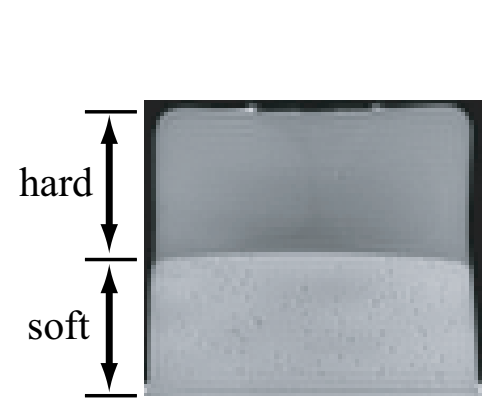

T2-weighted image

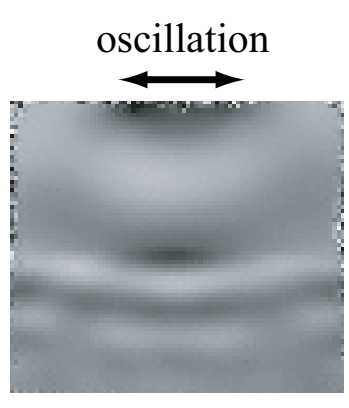

MR Elastography
Figure 10. MRE of hard and soft material. The wavelength in the hard part (top half) is longer than that in the soft part (bottom half).

hydrogel phantom obtained with application of $125 \mathrm{~Hz}$ and $250 \mathrm{~Hz}$ transverse waves. These images illustrate that the wavelength becomes half when the frequency is doubled.

Another controllable parameter in MRE is the phase of the MSG in relation to the oscillation. In MRE method, MSG is synchronized to the external oscillations. However, the phase difference between them can be altered by changing the delay time to start MSG from the application of the external oscillation (Figure 5). By acquiring multiple images with gradually increasing the phase offsets, we can ob-

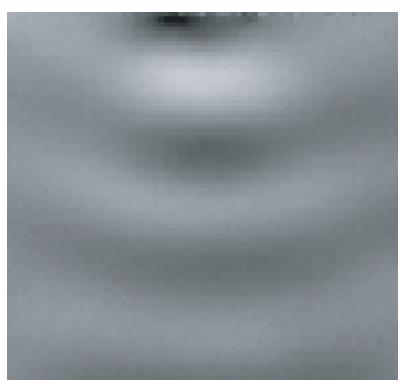

$125 \mathrm{~Hz}$

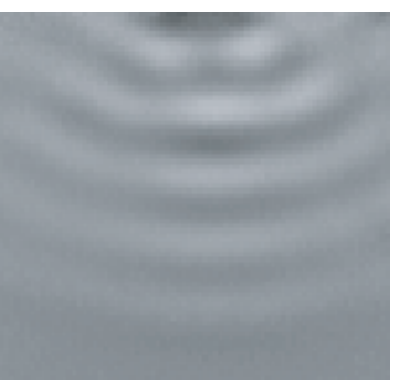

$250 \mathrm{~Hz}$
Figure 11. The relationship between frequency of oscillation and wavelength. The wavelength is in inverse proportion to the frequency in the same material.

tain a series of images, from which we can easily recognize the propagation of the strain waves. MRE images acquired with multiple phase offsets using 10\% - 5\% two layer PVA hydrogel phantom are shown in Figure 12. These images were acquired with application of $125 \mathrm{~Hz}$ transverse waves and the delay time was advanced by $1 \mathrm{~ms}$ for each image. Therefore, phase offset increases every $1 / 8$ cycle of the oscillation. From these images, we can observe the strain waves traveling downward.

Finally, we show an in vivo example of MRE. T2weighted image and MRE image obtained with application of $100 \mathrm{~Hz}$ transverse waves to human calf are shown in Figure 13. From the MRE image, we can observe the strain waves near the surface only. This implies that the strain waves are rapidly attenuating. There are only a few reports on in vivo MRE studies. Brain studies were reported by Manduca et al. [10,11]. A bite-block driver is used in these studies. Muscle studies are reported by Sacka et al. [12] and Heers et al. [13]. These studies are performed by probe-type oscillator.

MRE method is effective for measuring the elasticity of human tissue in vivo, however, it is necessary to develop an oscillator which can supply effective energy into the deeper part of the human body in order to measure the elasticity of the internal organs. On the other hand, a static MRE method is reported [14]. In this method, not the dynamic stress, but the static stress is applied to a material. Deformation induced by static stress is measured with stimulated echo imaging and the elasticity is calculated. This method may be suitable for measuring the elasticity of the internal organs at a deeper part of human body. 


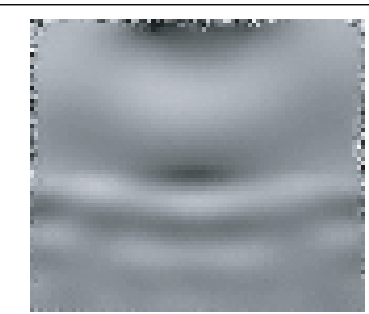

0

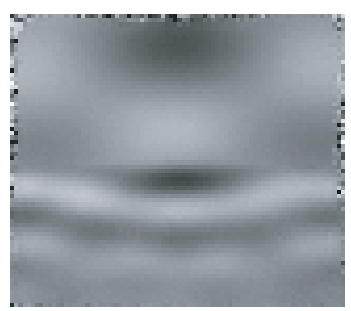

$2 \pi / 4$

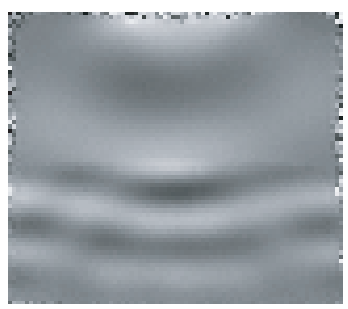

$4 \pi / 4$

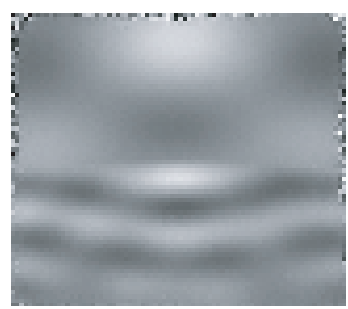

$6 \pi / 4$

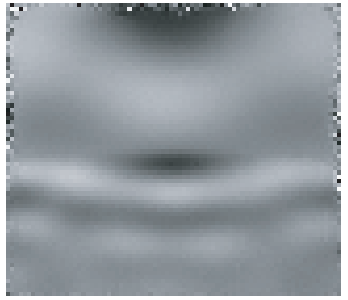

$\pi / 4$

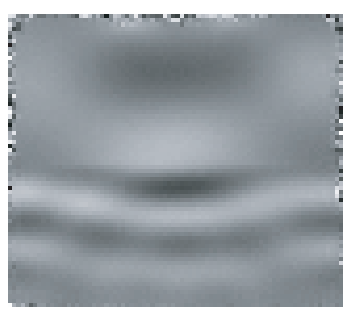

$3 \pi / 4$

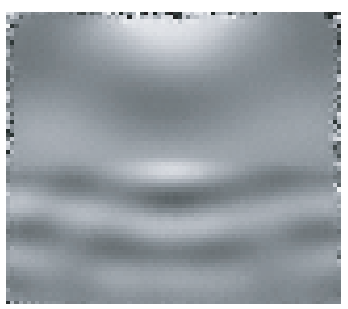

$5 \pi / 4$

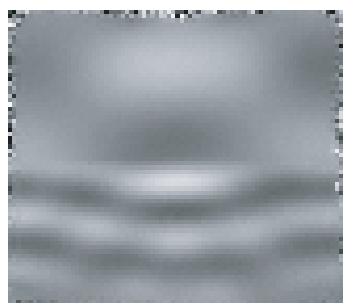

$7 \pi / 4$
Figure 12. Phase of oscillation and MRE. When the phase of oscillation is advanced, the wave propagation can be visualized in MRE images.

\section{Calculation of the elasticity from MRE im- ages}

By estimating the wavelength of the strain waves from the acquired MRE images, we can calculate the elasticity with eq.(4) and eq.(5). Thus, the correctness of the calculated elasticity depends on the accuracy of the wavelength estimation.

Recently, several methods to estimate wavelength from MRE images are reported. One is the local frequency estimation (LFE) algorithm based on the log-normal quadra-

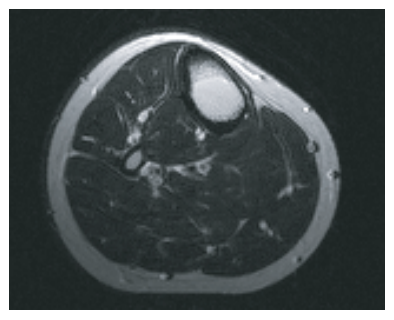

T2-weighted image

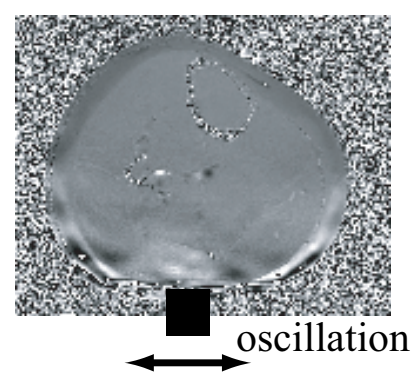

MR Elastography

\section{Figure 13. MRE images of human calf. The wave propagation can be observed only in the surface area.}

ture wavelet filters proposed by Manduca et al. [15], and the other is the instantaneous frequency method that uses the Hilbert transform (IFHT method) proposed by Oshiro et al. [16-18]. Since the MRE images contain a large amount of noise originating from the additional electric equipments of the system, however it is difficult to estimate wavelength accurately. To cope with this problem, IFHT method simply applies a moving average filter to the MRE images for reduction of the noise. However, the spatial resolution of the estimating wavelength becomes low because of application of the filter. An inverse approach was proposed by Manduca et al. [19]. This method reconstructs elasticity and attenuation maps in the strain waves of MRE images. Though this method provides better resolution for the reconstruction of stiffness images, it is very sensitive to the noise in comparison to the LFE.

In contrast to the methods utilizing single image described above, the use of multiple images with different phase offsets potentially improve the accuracy and the spatial resolution of the wavelength estimation. Suga et al. proposed a multiple-phase patchwork offsets method [20,21]. This method can estimate the wavelength with higher spatial resolution in comparison to the methods of utilizing single image. However, there is a limitation that the number of images is restricted by the wavelength. To overcome this limitation, we proposed an elastic wave fitting method [22]. This method have a feature that the number of images is unlimited and can use arbitrary spatial resolution. Therefore, one can choose either the robustness against noise or higher resolution of estimation.

Though it is important to cope with the reflection and the refraction as mentioned in the section 4 . One is a method called spatio-temporal directional filtering which was proposed by Manduca et al. [11,23]. This method uses directional filter which separates several waves caused by the re- 
flection and the refraction.

\section{Conclusion}

In this paper, we surveyed MRE method, which is a novel noninvasive method for measuring the elasticity of a material. We explained the relationship between the elasticity of a material and the velocity of the strain waves. Then we verified this relationship with several real MRE images.

MRE method is new and it still requires some improvements for the clinical use. One of the target is to develop an effective oscillator which can propagate the strain waves into the deep and wide part of a material. Progress of accurate method to estimate wavelength is another potential target. By improving these points, it will be an important method for the clinical studies.

\section{Acknowledgment}

This paper is supported in part by Informatics Research Center for Development of Knowledge Society Infrastructure (COE program of the Ministry of Education, Culture, Sports, Science and Technology, Japan) and in part by the Ministry of Education, Culture, Sports, Science and Technology of Japan under the Grant in Aid for Scientific Research 14570854.

\section{References}

[1] Muthipillai R, Lomas DJ, Rossman PJ, Greenleaf JF, Manduca A, Ridderer SJ, Ehman RL. Magnetic resonance imaging of acoustic strain waves. Proc Sec Magn Reson Nice, 1:189, 1995.

[2] Muthipillai R, Lomas DJ, Rossman PJ, Greenleaf JF, Manduca A, Ehman RL. Magnetic resonance elastography by direct visualization of propagating acoustic strain waves. Science, 269:1854-1857, 1995.

[3] Muthpillai R, Ehman RJ. Magnetic resonance elastography. Nature Medicine, 2:601-603, 1996.

[4] Muthipillai R, Rossman PJ, Lomas DJ, Greenleaf JF, Ridderer SJ, Ehman RL. Magnetic resonance imaging of transverse acoustic strain waves. Magn Reson Med, 36:266-274, 1996.

[5] Bishop J, Poole G, Leitch M, Plewes DB. Magnetic resonance imaging of shear wave propagation in excised tissue. J Magn Reson Imaging, 8:1257-1265, 1998.

[6] Nakagawa T. Rheology (in Japanese). 12-37, Iwanami shoten, Tokyo, 1978

[7] Nakamura K. Foundation of Rheology (in Japanese). 7-47, Sangyo tosho, Tokyo, 1991

[8] van Dijk P. Direct cardiac NMR imaging of heart wall and blood flow velocity. J Comput Assist Tomogr, 8:429-436, 1984.
[9] Moran PR, Moran RA, Karstaedt N. Verification and evaluation of internal flow and motion. True magnetic resonance imageing by the phase gradient modulation method. Radiology, 154:433-441, 1985.

[10] Manduca A, Oliphant TE, Dresner MA, Mahowald JL, Kruse SA, Amromin E, Felmlee JP, Greenleaf JF, Ehman RL. Magnetic resonance elastography: Non-invasive mapping of tissue elasticity. Medical Image Analysis, 5:237-254, 2001.

[11] Manduca A, Lake DS, Kruse SA, Ehman RL. Spatiotemporal directional filtering for improved inversion of MR elastography images. Medical Image Analysis, 7:465-473, 2003

[12] Sacka I, Bernarding J, Braun J Analysis of wave patterns in MR elastography of skeletal muscle using coupled harmonic oscillator simulations. Magnetic Resonance Imaging, 20:95-104, 2002.

[13] Heers G, Jenkyn T, M. Dresner A, Klein MO, Basford JR, Kaufman KR, Ehman RL, An KN. Measurement of muscle activity with magnetic resonance elastography. Clinical Biomechanics, 18:537-542, 2003.

[14] Chenevert TL, Skovoroda AR, O’Donnell M, Emelianov SY. Elasticity reconstructive imaging by means of stimulated echo MRI. Magn Reson Med, 39:482-490, 1998.

[15] Manduca A, Muthupillai R, Rossman PJ, Greenleaf JF, Ehman RL. Image Processing for Magnetic Resonance Elastography. Proc. of the SPIE, 616-623, 1996.

[16] Oshiro O, Suga M, Minato K, Okamoto J, Takizawa O, Matsuda T, Komori M, Takahashi T. Elasticity analysis by MR elastography using the instantaneous frequency method. (in Japanese) medical imaging technology Japan 18:158-166, 2000

[17] Boashash B. Estimating and interpreting the instantaneous frequency of a signal - part 1 : Fandamentals. Proc. of the IEEE, 80(4):520-538, 1992.

[18] Boashash B. Estimating and interpreting the instantaneous frequency of a signal - part 2: Algorithms and applications. Proc. of the IEEE, 80(4):539-568, 1992.

[19] Manduca A, Dutt V, Borup DT, Muthupillai R, Ehman RL, Greenleaf JF. Reconstruction of Elasticity and Attenuation Maps in Shear Wave Imaging: An Inverse Approach. MICCAI 98, 606-613, 1998.

[20] Suga M, Matsuda T, Minato K, Oshiro O, Chihara K, Okamoto J, Takizawa O, Komori M, Takahashi T. Measurement of In-vivo Local Shear Modulus by Combining Multiple Phase Offsets. MEDINFO2001 933-937, 2001

[21] Suga M, Matsuda T, Minato K, Oshiro O, Chihara K, Okamoto J, Takizawa O, Komori M, Takahashi T. Measurement of In Vivo Local Shear Modulus Using MR Elastography Multiple-Phase Patchwork Offsets. Trans. of IEEE on BIOMEDICAL ENGINEERING 50(7):908-915, 2003

[22] Oida T, Ohno T, Amano A, Matsuda T, Kang Y, Azuma T, Tsutsumi S, Okamoto J, Takizawa O. Estimation of Viscoelastic Properties from Multiple Phase MR Elastography using Elastic Wave Fitting Method. (in Japanese) Technical Report of IEICE MI2003-19:43-48, 2003

[23] Manduca A, Lake DS, Ehman RL. Spatio-temporal Directional Filtering for Improved Inversion of MR Elastography Images. MICCAI 2002, 293-299, 2002 